\title{
Recent brain function measurement
}

\author{
Toshiyuki Tanaka \\ Faculty of Science and Technology, Keio University
}

Recently, the brain function has been measured by some imaging systems. The imaging systems diagnose several kind of diseases, and also check the recovery level of rehabilitation. The following systems are well known worldwide as the imaging systems.

(1) EEG (Electroencephalogram)

(2) MEG (Magnetic encephalogram)

(3) SPECT (Single photon emission computed tomography)

(4) PET (Positron emission tomography)

(5) fMRI (functional magnetic resonance imaging)

(6) fNIRS (functional near-infrared spectroscopy)

The above systems measure the different biological reaction. EEG and MEG measure the neural activity in the brain, and others (SPECT, PET, fMRI and fNIRS) measure the change of regional cerebral blood volume. The fNIRS has been frequently used in the brain function measurement at the rehabilitation, for the reason that the system can be used with metal-containing devices.

We have shown successful results by the fNIRS and fMRI in some conferences. Although the EEG and MEG have been not frequently used for the brain function analysis, because the analysis is complicate and the computer-based implementation is difficult and does not have sufficient results. We think that the EEG and MEG are more suitable for brain function measurement, since the systems directly measure the neural activity in the brain. Professor Takaaki Nara with University of Tokyo has proposed the mathematical researches of activation site in brain using MEG and EEG, and the study receives attention from the brain function researchers. We think that the brain function is analyzed by using computer, based on our research achievement. The diagnosis by EEG shows the recovery condition of brain function from the view of neural activity. 